\title{
Melanoma-Associated Antigen Family
}

National Cancer Institute

\section{Source}

National Cancer Institute. Melanoma-Associated Antigen Family. NCI Thesaurus. Code C113841.

A family of proteins that were first detected in male germ cells, placenta and various tumors that were orig inally not found in normal tissues. Their genes are located in four clusters on the X chromosome. All of the members of the family share a stretch of about 200 amino acids, called the MAGE conserved domain, which is usually located near the Cterminal end of the protein and may allow MAGE proteins to interact with cellular receptors. For most members their entire coding sequences are located in the last exon of their respective genes. 